\title{
Servidores Web de Altas Prestaciones
}

\author{
Pedro A. Castillo, Jose L. Bernier, Antonio M. Mora, \\ Pablo García-Sánchez, M. G. Arenas \\ Departamento de Arquitectura y Tecnología de Computadores. \\ Universidad de Granada \\ Granada, España \\ pedro@atc.ugr.es
}

\begin{abstract}
Resumen. Para crear un sitio web exitoso debemos ser cuidadosos con muchos detalles, ya que la arquitectura, la selección del hardware, o la implementación de las aplicaciones afectarán al rendimiento del sistema completo. Es más, un sitio que no esté preparado para dar servicio a un número creciente de clientes o usuarios, a la larga puede llevar al fracaso a la empresa. En este trabajo, se presenta una visión general de la nueva asignatura Servidores Web de Altas Prestaciones, del nuevo plan de estudios del Grado en Ingeniería Informática de la Universidad de Granada, así como la metodología a seguir para impartir tanto la teoría como las prácticas. A través de un aprendizaje principalmente práctico tratamos de que el alumno descubra cómo diseñar, tanto a nivel de hardware de red como de configuración de software, un servidor web de altas prestaciones. A lo largo de la asignatura pretendemos dar una visión detallada de los conceptos y herramientas utilizadas para crear o mantener la infraestructura de un sistema de forma adecuada. Estudiaremos las ideas y prácticas para asegurar la escalabilidad del sistema, asegurando el servicio a nuevos usuarios y manteniendo el sistema activo.
\end{abstract}

Palabras Clave: servidor web, disponibilidad, balanceo de carga, docencia

\begin{abstract}
To create a successful website, we must be careful with many details, as the architecture, hardware selection, and implementation of applications, and how they affect the performance of the entire system. Moreover, a site that is not prepared to serve a growing number of customers or users, can eventually lead to failure of the company. In this paper, we present an overview of the new subject "High Performance Web Servers" in the new curriculum's Degree in Computer Engineering from the University of Granada, as well as the methodology to teach both theory and practice. Through a practical learning, we try to make the student learn how to design both the network hardware and software configuration of a high performance web server. Throughout the course we intend to give a detailed overview of the concepts and tools used to create and maintain the infrastructure of a system properly. We will study the ideas and practices to ensure the scalability of the system and the service to new users.
\end{abstract}

Keywords: web server, availability, load balancing, teaching 


\section{Introdución}

Al crear un sitio web, los administradores de sistemas tienen por delante un duro trabajo inicial para hacer más fácil el mantenimiento posterior.

Como primera y más fácil aproximación, lo habitual es montar un servidor en una sola máquina. Esto, que resulta fácil y rápido, a la larga necesitará mucho más trabajo de mantenimiento, y probablemente haga que el sitio web experimente graves problemas. Las tareas derivadas de los problemas de hardware y software, así como dar servicio a diferentes cargas de trabajo, acaban siendo un trabajo demasiado costoso en servidores sencillos que deben atender gran tráfico [1].

La única solución a estos problemas pasa por transformar el sistema en una granja web (web farm) compuesta de varios servidores dedicados cada uno a una tarea concreta. Una granja web es un sistema escalable y con alta disponibilidad. Puede resultar mucho más complejo y trabajoso instalarlo y configurarlo, pero es el tipo de sistema que puede soportar un alto tráfico de red y dar servicio a millones de usuarios $[2,5]$.

Del conjunto de servidores dedicados a servir contenido web a los usuarios finales, algunos pueden servir contenido HTML estático, mientras que otros pueden albergar las bases de datos, o bien ofrecer contenido dinámico. Se trata de sistemas complejos, pero muy flexibles a la hora de diseñarlos y construirlos.

A pesar de la importancia de estos temas, en gran parte de las titulaciones de Ingeniería Informática existía una carencia en cuanto a que ciertos temas referentes a tecnologías web no se estudiaban.

En octubre de 2010 comenzó la implantación de los estudios del nuevo Grado en Ingeniería Informática por la Universidad de Granada. Como consecuencia de la visión de las necesidades del mercado laboral, y teniendo en cuenta el Libro Blanco de la titulación y los recursos humanos y materiales disponibles, se decidió proponer un único título de Grado en Ingeniería Informática, con cinco especialidades profesionales diferenciadas y elevado atractivo en el entorno socioeconómico, regional, nacional y europeo. Las especialidades son:

- Computación y Sistemas Inteligentes

- Ingeniería de Software

- Ingeniería de Computadores

- Sistemas de Información

- Tecnologías de la Información

Los especialistas en Tecnologías de la Información son los encargados de configurar, mantener, actualizar, y garantizar el funcionamiento seguro de las plataformas de cómputo en las empresas. Concretamente, algunos de los temas de estudio de esta última especialidad son la consolidación de servidores a través de técnicas de virtualización o las posibilidades que abre el Cloud Computing [6,7]. 
Una de las asignaturas obligatorias de la especialidad de Tecnologías de la Información es Servidores Web de Altas Prestaciones.

Esta asignatura tiene como objetivo principal que el alumno aprenda a diseñar, instalar y configurar un servidor de altas prestaciones a partir de las necesidades de un cliente. Los contenidos generales de la asignatura son los siguientes: evaluación de necesidades; requisitos hardware para servidores; criterios de coste, calidad y prestaciones; seguridad en servidores; prestaciones (tolerancia a fallos, alta disponibilidad, etc); ejemplos y aplicaciones.

Con estos contenidos se pretende identificar los problemas más habituales relativos a las aplicaciones, servidores, red y arquitectura, de servidores mal planificados o mal configurados.

En cuanto a los objetivos formativos de la asignatura y los distintos niveles de profundización, capacidades y destrezas que debe adquirir un alumno tras la superación de la asignatura, expresados como resultados esperables de la enseñanza, cabe destacar los siguientes:

- Explicar y exponer los principales conceptos relacionados con la alta disponibilidad, redundancia y tolerancia a fallos.

- Determinar posibles problemas de escalabilidad de una instalación.

- Configurar un balanceador de carga de forma adecuada a las necesidades.

- Realizar las tareas de la administración de un sistema de alta disponibilidad.

- Señalar y describir las tecnologías hardware actuales para la instalación de granjas o agrupaciones de servidores.

- Encontrar y aprovechar las soluciones idóneas para servidores de alta disponibilidad.

- Estimar los costes de diseño, montaje, configuración, mantenimiento y operación del sistema.

- Seleccionar diferentes herramientas de seguridad y describir su uso.

- Establecer la configuración de los servidores y las estrategias para optimizar la seguridad del sistema.

- Establecer políticas de calidad y prestaciones del sistema.

- Seleccionar, instalar y usar las herramientas de análisis y monitorización de las prestaciones del sistema.

- Diseñar y configurar un sistema web de alta disponibilidad.

- Diseñar un plan de copias de seguridad y recuperación para resolver cualquier problema en el sistema.

Como competencias específicas de la asignatura se establecieron:

- TI2. Capacidad para seleccionar, diseñar, desplegar, integrar, evaluar, construir, gestionar, explotar y mantener las tecnologías de hardware, software y redes, dentro de los parámetros de coste y calidad adecuados.

- TI6. Capacidad de concebir sistemas, aplicaciones y servicios basados en tecnologías de red, incluyendo Internet, web, comercio electrónico, multimedia, servicios interactivos y computación móvil. 
- $\quad$ TI7. Capacidad para comprender, aplicar y gestionar la garantía y seguridad de los sistemas informáticos.

El resto del trabajo está organizado como sigue: la Sección 2 justifica la necesidad de la asignatura en el plan de estudios. En la Sección 3 se presentan los temarios de teoría y prácticas, seguido de la metodología docente a seguir (Sección 4). Finalmente se detalla la estrategia de evaluación en la Sección 5, para terminar con unas conclusiones en la Sección 6.

\section{Justificación de la necesidad de la asignatura en el Grado de Informática}

Cuando nos encargan el desarrollo de un sitio web, en muchas ocasiones comenzamos el desarrollo sobre una máquina que hará todas las tareas necesarias (servidor web, almacenamiento, bases de datos, etc). Es la opción más rápida, y si no hay muchos usuarios o el tráfico es bajo, suele funcionar.

Este tipo de sistemas sólo necesita una máquina con el sistema operativo configurado (ya sea, Linux, Windows, OS X o Solaris) una aplicación servidor-web (como Apache, nginx o IIS) y una conexión a internet. Estos sistemas resultan muy baratos inicialmente, por lo que muchas empresas optan por esta configuración. Además, como decimos, es suficiente para sistemas muy pequeños con poco tráfico.

En la misma máquina se instala el software necesario para el servidor web, servidor de mensajería, la base de datos y el almacenamiento de ficheros. A pesar del bajo coste y de que todo vaya bien en la fase de desarrollo y pruebas (por el bajo tráfico), ese servidor puesto en producción puede acabar experimentando diversos problemas.

De hecho, cuando los cambios se vuelven inmanejables, no queda más remedio que reestructurar todo el sistema para configurar una arquitectura basada en granja web (ver Figura 1) que nos permita dar el servicio esperado y sea fácil de mantener.

No merecerá la pena comenzar a parchear el sistema en varios niveles, invirtiendo tiempo, recursos humanos e incluso dinero en adquirir hardware más nuevo y potente: tratar durante meses de adaptar o retocar el sistema para que siga funcionando e intente dar el servicio que debe, no valdrá la pena, ya que saldrá más caro que rediseñar un buen sistema basado en una granja web.

Para ello, y como en casi todo sistema informático, hay que definir correctamente los requisitos del sitio web a desarrollar, invertir el dinero necesario de forma efectiva, y planificar las tareas de configuración y desarrollo de las aplicaciones y servicios de forma adecuada. 
En esta asignatura estudiaremos los principales problemas en la construcción y configuración de este tipo de sistemas: problemas de la arquitectura del sistema, con la disponibilidad y con la carga del sistema, principalmente. Asimismo, daremos una visión detallada de los conceptos y herramientas utilizadas para crear o mantener la infraestructura de un sistema de forma adecuada. Estudiaremos las ideas y prácticas para asegurar la escalabilidad del sistema, asegurando el servicio a nuevos usuarios, así como para mantener el sistema activo y dando servicio el máximo tiempo posible.

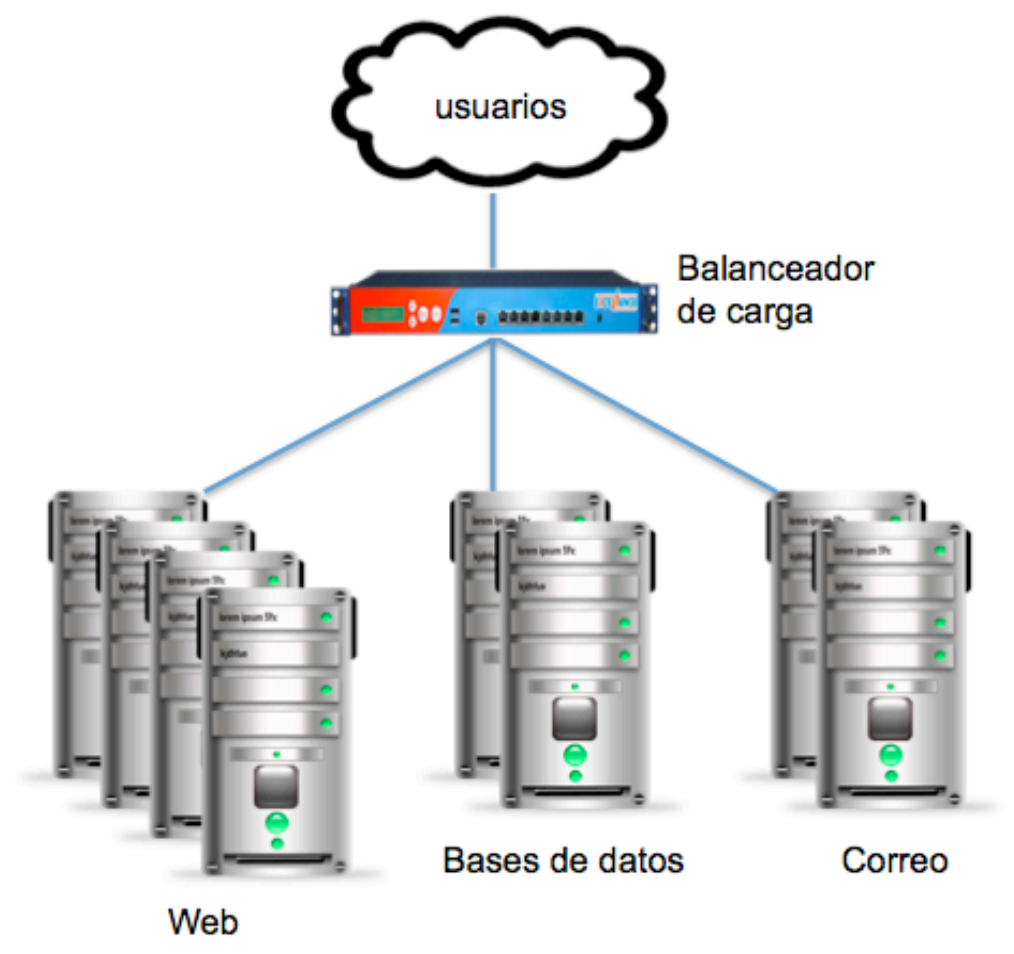

Figura 1. Estructura general de una granja web.

\section{Temarios de teoría y prácticas}

De acuerdo con los objetivos descritos, se ha planteado el siguiente temario para la asignatura:

- El Tema 1 "Introducción" se dedicará al estudio de los problemas que podemos experimentar al montar un sitio web que tendrá muchos usuarios y un alto tráfico en una máquina sola. De esta forma justificaremos la necesidad de montar granjas web en estos casos.

- $\quad$ El Tema 2 "Alta disponibilidad y escalabilidad en servidores web" presentará los conceptos de alta disponibilidad, escalabilidad y tolerancia a fallos en 
general. El objetivo siempre es crear sistemas que den servicio las $24 \mathrm{~h}$ con el mínimo de errores.

- $\quad$ El Tema 3 "La red de una granja web" presenta diferentes arquitecturas de red para crear una granja web, siempre con los objetivos de rendimiento, disponibilidad y facilidad de mantenimiento [1].

- El Tema 4 "Balanceo de carga" detalla diversas opciones de balanceo de carga, tanto software como hardware $[2,5]$.

- El Tema 5 "Medición de prestaciones" presenta algunas consideraciones sobre cómo evaluar las prestaciones de un sistema web de altas prestaciones.

- El Tema 6 "Técnicas de seguridad" presenta los diferentes problemas de seguridad a tener en cuenta para asegurar los servidores. Se comentan las prácticas comunes para resolver problemas de seguridad, el uso de cortafuegos, protección de servidores y políticas de seguridad.

- En el Tema 7 "Requisitos hardware de servidores web" se estudian los principales requisitos a nivel de hardware para la construcción $y$ configuración de estos sistemas

- De forma similar, el Tema 8 "Requisitos software de servidores web" estudia los requisitos a nivel de software.

- En el Tema 9 "Bases de datos de alta disponibilidad" se detallan las cuestiones principales relativas a la configuración de bases de datos en sistemas de alta disponibilidad [4].

- Por último, el Tema 10 "Aplicaciones y ejemplos" presenta diferentes sistemas usados en empresas u organizaciones en las que se hace uso de este tipo de sistemas.

Igualmente, se han propuesto, como complemento docente, los siguientes seminarios:

- Seminario práctico 1: Componentes y configuración hardware de un cluster web

- Seminario práctico 2: Alternativas de sistemas de balanceo de carga

- Seminario práctico 3: Alternativas para clustering en servidores web

- Seminario práctico 4: Configuración de sistemas y técnicas de seguridad en servidores web

- Seminario práctico 5: Alternativas en bases de datos de altas prestaciones

Finalmente, la parte práctica de la asignatura está compuesta por las siguientes prácticas: 
- Práctica 1: Presentación de las prácticas y preparación de las herramientas

- Práctica 2: Replicación de contenidos entre servidores

- Práctica 3: Balanceo de carga y clustering en servidores web

- Práctica 4: Evaluación de prestaciones

- Práctica 5: Uso de bases de datos en sistemas de alta disponibilidad

En las siguientes secciones se presenta de forma más detallada la metodología prevista para el desarrollo de la asignatura (teoría y prácticas), así como la estrategia de evaluación.

\section{Metodología propuesta}

El desarrollo de la asignatura se basará en los siguientes tipos de actividades de formación:

- Clases teóricas para la presentación en el aula de los conceptos propios de la materia, haciendo uso de metodología expositiva con lecciones magistrales participativas y medios audiovisuales.

- Clases prácticas, a través de las cuales se pretende mostrar al alumnado cómo debe actuar a partir de la aplicación de los conocimientos adquiridos.

- Seminarios para tratar en profundidad temáticas relacionadas con la materia. Se trata de actividades basadas en la indagación, el debate, la reflexión y el intercambio.

- Estudio y trabajo en grupo: Se trata de trabajos propuestos por el profesor, a través de los cuales y de forma grupal se profundiza en aspectos concretos de la materia, posibilitando a los estudiantes avanzar en la adquisición de determinados conocimientos y procedimientos de la materia.

Las actividades formativas propuestas más arriba se desarrollarán desde una metodología participativa y aplicada que se centra en el trabajo del estudiante (presencial y no presencial/individual y grupal). Las clases teóricas, los seminarios, las clases prácticas, las tutorías, el estudio y trabajo autónomo y el grupal son las maneras de organizar los procesos de enseñanza y aprendizaje.

\subsection{Herramientas utilizadas en la asignatura}

Un aspecto importante son las herramientas utilizadas en el desarrollo de la asignatura. En este sentido, un aspecto fomentado es la creación de blogs de alumnos, en los que se describan las inquietudes, intereses, enlaces y trabajos de los mismos en relación a la asignatura. Para ello, se insta a que éstos hagan la entrega de sus prácticas a modo de página web, consiguiendo igualmente un formato legible y bien presentado en cualquier sistema. Esta medida (probada anteriormente en otras 
asignaturas) suele tener un gran acogimiento entre los alumnos, manteniendo muchos de los cuales dicho blog en adelante.

La otra herramienta utilizada es SWAD: Sistema Web de Apoyo a la Docencia(http://swad.ugr.es) [3], una plataforma libre de teleformación desarrollada y utilizada en la Universidad de Granada en los últimos 12 cursos académicos.

SWAD integra diversas funciones de apoyo al aprendizaje, a la docencia y a la gestión de los datos de los estudiantes. Entre ellas el acceso a información sobre las asignaturas (guía docente, horarios, bibliografía,...), la descarga de documentos (transparencias, relaciones de problemas), las listas y fichas de alumnos y profesores, los foros de discusión, la asignación de actividades, la autoevaluación mediante exámenes interactivos o la consulta individual de calificaciones. En dicha plataforma se hace el seguimiento de los alumnos, la publicación de los temarios y guiones de prácticas, la entrega de ejercicios, trabajos y prácticas, y la publicación (con consulta individual) de las calificaciones.

\section{Evaluación}

Con objeto de evaluar la adquisición de los contenidos y competencias, se utilizará un sistema de evaluación diversificado, seleccionando las técnicas de evaluación más adecuadas para cada materia o asignatura en cada momento, que permita poner de manifiesto los diferentes conocimientos y capacidades adquiridos por el alumnado al cursar la materia.

Se utilizarán las siguientes técnicas de evaluación:

- Para la parte teórica se realizará un examen final tipo test así como entregas de ejercicios y resultados de las actividades propuestas.

- Para la parte práctica se realizarán prácticas de laboratorio y desarrollo de proyectos (individuales o en grupo), y se valorarán las entregas de los informes/memorias realizados por los alumnos.

- En cuanto a los seminarios, estos se evaluarán teniendo en cuenta la asistencia y participación.

- En cuanto a la parte de trabajo en grupo, se evaluará teniendo en cuenta la presentación oral así como la memoria del trabajo desarrollado.

El resultado de la evaluación será una calificación numérica obtenida mediante la suma ponderada de las calificaciones correspondientes a la parte teórica, la parte práctica y una parte relacionada con el trabajo autónomo de los alumnos, los seminarios impartidos y el aprendizaje basado en proyectos. 


\section{Conclusiones}

El objetivo de este trabajo es la presentación de la asignatura Servidores Web de Altas Prestaciones, de la especialidad Tecnologías de la Información del nuevo plan de estudios del Grado en Ingeniería Informática de la Universidad de Granada.

La metodología docente está pensada para impartir la asignatura de forma práctica siempre que sea posible. En cuanto a las herramientas docentes que se usarán, se proponen SWAD, una plataforma web para facilitar las tareas de docencia, y diversos blogs de alumnos que versen sobre la asignatura y sus contenidos. Los años de experiencia usando estas herramientas en otras asignaturas y la metodología propuesta han demostrado que el método funciona, y que los alumnos adquieren y afianzan conocimientos.

Por último, por su temática, cabe destacar la necesidad de mantener actualizados los temarios de teoría y prácticas con las últimas tecnologías, herramientas y metodologías de estudio en la construcción y configuración de servidores web de altas prestaciones.

\section{Agradecimientos}

Los autores agradecen a Mario Barchéin sus aportaciones y ayuda en la preparación de la asignatura.

\section{Referencias}

[1] B. Bloom. Deploying and Managing Microsoft .NET Web Farms. ISBN 0672320576. Sams White Bks. Indianapolis, IN, USA. 2001

[2] T. Bourke. Server Load Balancing. 1 Edición. ISBN 0-596-00050-2. O'Reilly \& Associates, Inc., 101 Morris Street, Sebastopol, CA 95472, USA. 2001

[3] A. Cañas, D. J. Calandria, E. M. Ortigosa, E. Ros, A. F. Díaz. SWAD: Web System for Education Support. In B. Fernández-Manjón, J. M. Sánchez Pérez, J. A. GómezPulido, M. A. Vega Rodríguez, J. Bravo-Rodríguez (Eds.): Computers And Education: E-learning - from Theory to Practice, 241 pages, Chapter 12, pp. 133142, ISBN 978-1-4020-4913-2, Springer, 2007.

[4] A. Davies. High Availability MySQL Cookbook. ISBN 978-1-847199-94-2. Packt Publishing. 2010

[5] C. Kopparapu. Load Balancing Servers, Firewalls, and Caches. ISBN 0-47141550-2. Wiley Computer Publishing John Wiley \& Sons, Inc. USA. 2001. 
[6] M. Miller. Cloud Computing: Web-Based Applications That Change the Way You Work and Collaborate Online. ISBN-10: 0789738031. ISBN-13: 978-0789738035. $1^{\mathrm{a}}$ Edición. QUE. 2012

[7] B. Furht and A. Escalante. Handbook of Cloud Computing. Google eBook. ISBN: 978-1-4419-6523-3. Springer. 2010 